\title{
Conservation Laws for a Variable Coefficient Variant Boussinesq System
}

\author{
Ben Muatjetjeja and Chaudry Masood Khalique \\ Department of Mathematical Sciences, International Institute for Symmetry Analysis and Mathematical Modelling, \\ North-West University, Mafikeng Campus, Private Bag X 2046, Mmabatho 2735, South Africa
}

Correspondence should be addressed to Chaudry Masood Khalique; masood.khalique@nwu.ac.za

Received 21 November 2013; Accepted 6 January 2014; Published 12 February 2014

Academic Editor: Hossein Jafari

Copyright ( 2014 B. Muatjetjeja and C. M. Khalique. This is an open access article distributed under the Creative Commons Attribution License, which permits unrestricted use, distribution, and reproduction in any medium, provided the original work is properly cited.

We construct the conservation laws for a variable coefficient variant Boussinesq system, which is a third-order system of two partial differential equations. This system does not have a Lagrangian and so we transform it to a system of fourth-order, which admits a Lagrangian. Noether's approach is then utilized to obtain the conservation laws. Lastly, the conservation laws are presented in terms of the original variables. Infinite numbers of both local and nonlocal conserved quantities are derived for the underlying system.

\section{Introduction}

The first type of variant Boussinesq equations $[1,2]$ is given by

$$
\begin{gathered}
u_{t}+v_{x}+u u_{x}=0, \\
v_{t}+(u v)_{x}+u_{x x x}=0,
\end{gathered}
$$

and was introduced as a model for water waves [3]. Wang in his paper [4] obtained the solitary wave solutions of ((1a) and (1b)) by using homogeneous balance method. The periodic wave solutions of ((1a) and (1b)) were derived in [5] by using ansatz method and the multisolitary wave solutions were obtained in [6] using the homogeneous balance method. Xu et al. [7] obtained traveling wave solutions of ((1a) and (1b)). Conservation laws for ((1a) and (1b)) were derived in [8].

Conservation laws play a vital role in the solution process of differential equations (DEs) because they describe physical properties that remain constant throughout the various processes that occur in the physical world. Thus it is very important to compute conservation laws for differential equations. One can see from the various published papers (see, e.g., [911]) that conservation laws have been used in studying the existence, uniqueness, and stability of solutions of nonlinear partial differential equations. They have also been applied in the development and use of numerical methods (see, e.g.,
$[12,13])$. Most importantly, conserved vectors associated with Lie point symmetries have been used to derive exact solutions of some partial differential equations [14-16].

In this paper, we study the variable coefficient variant Boussinesq system:

$$
\begin{gathered}
u_{t}+\alpha(t) v_{x}+\beta(t) u u_{x}=0, \\
v_{t}+\beta(t)(u v)_{x}+\psi(t) u_{x x x}=0,
\end{gathered}
$$

which generalizes the system ((1a) and (1b)). In ((2a) and $(2 \mathrm{~b})), \alpha(t), \beta(t)$, and $\psi(t)$ are arbitrary functions of $t$, with $\psi(t)$ describing the different diffusion strength, $u=u(x, t)$ representing the field of a horizontal velocity, and $v=v(x, t)$ representing the amplitude describing the deviation from the equilibrium position of the liquid.

The objective of the present study is to construct conservation laws for the system $((2 a)$ and $(2 b))$.

The paper is organized as follows. In Section 2 we briefly give the preliminaries concerning the Noether symmetry approach. Section 3 obtains the conservation laws for the system $((2 \mathrm{a})$ and $(2 \mathrm{~b}))$. Finally, in Section 4 concluding remarks are presented. 


\section{Preliminaries}

Here we present some salient features of Noether operators concerning the system of two partial differential equations. These results will be utilized in Section 3. The reader is referred to $[8,17-19]$ for further details.

Consider the vector field

$X=\xi^{1}(t, x, U, V) \frac{\partial}{\partial t}+\xi^{2}(t, x, U, V) \frac{\partial}{\partial x}+\eta^{1}(t, x, U, V) \frac{\partial}{\partial U}$

$$
+\eta^{2}(t, x, U, V) \frac{\partial}{\partial V}
$$

which has the second-order prolongation

$$
\begin{aligned}
X^{[2]}= & \xi^{1}(t, x, U, V) \frac{\partial}{\partial t}+\xi^{2}(t, x, U, V) \frac{\partial}{\partial y} \\
& +\eta^{1}(t, x, U, V) \frac{\partial}{\partial u}+\eta^{2}(t, x, U, V) \frac{\partial}{\partial V}+\zeta_{t}^{1} \frac{\partial}{\partial U_{t}} \\
& +\zeta_{t}^{2} \frac{\partial}{\partial V_{t}}+\zeta_{x}^{1} \frac{\partial}{\partial U_{x}}+\zeta_{x}^{2} \frac{\partial}{\partial V_{x}}+\zeta_{x x}^{1} \frac{\partial}{\partial U_{x x}}+\cdots,
\end{aligned}
$$

where

$$
\begin{aligned}
& \zeta_{t}^{1}=D_{t}\left(\eta^{1}\right)-U_{t} D_{t}\left(\xi^{1}\right)-U_{x} D_{t}\left(\xi^{2}\right), \\
& \zeta_{x}^{1}=D_{x}\left(\eta^{1}\right)-U_{t} D_{x}\left(\xi^{1}\right)-U_{x} D_{x}\left(\xi^{2}\right), \\
& \zeta_{t}^{2}=D_{t}\left(\eta^{2}\right)-V_{t} D_{t}\left(\xi^{1}\right)-V_{x} D_{t}\left(\xi^{2}\right), \\
& \zeta_{x}^{2}=D_{x}\left(\eta^{2}\right)-V_{t} D_{x}\left(\xi^{1}\right)-V_{x} D_{x}\left(\xi^{2}\right), \\
& \zeta_{x x}^{1}=D_{x}\left(\zeta_{x}^{1}\right)-U_{t} D_{x}\left(\xi^{1}\right)-U_{x} D_{x}\left(\xi^{2}\right),
\end{aligned}
$$

with

$$
\begin{aligned}
D_{t}= & \frac{\partial}{\partial t}+U_{t} \frac{\partial}{\partial U}+V_{t} \frac{\partial}{\partial V}+U_{t t} \frac{\partial}{\partial U_{t}}+V_{t t} \frac{\partial}{\partial V_{t}} \\
& +U_{x t} \frac{\partial}{\partial U_{x}}+V_{x t} \frac{\partial}{\partial V_{x}}+\cdots, \\
D_{x}= & \frac{\partial}{\partial x}+U_{x} \frac{\partial}{\partial U}+V_{x} \frac{\partial}{\partial V}+U_{x x} \frac{\partial}{\partial U_{x}}+V_{x x} \frac{\partial}{\partial V_{x}} \\
& +U_{x t} \frac{\partial}{\partial U_{t}}+V_{x t} \frac{\partial}{\partial V_{t}}+\cdots .
\end{aligned}
$$

$$
\begin{aligned}
\frac{\delta}{\delta V}= & \frac{\partial}{\partial V}-D_{t} \frac{\partial}{\partial V_{t}}-D_{x} \frac{\partial}{\partial V_{x}}+D_{t}^{2} \frac{\partial}{\partial V_{t t}}+D_{x}^{2} \frac{\partial}{\partial V_{x x}} \\
& +D_{x} D_{t} \frac{\partial}{\partial V_{x t}}-\cdots .
\end{aligned}
$$

Consider a system of two partial differential equations of two independent variables, $t$ and $x$, namely,

$$
\begin{aligned}
& E_{1}\left(t, x, U, V, U_{x}, V_{x}, U_{t}, V_{t}, U_{t t}, V_{t t}, U_{x x}, V_{x x}, \cdots\right)=0, \\
& E_{2}\left(t, x, U, V, U_{x}, V_{x}, U_{t}, V_{t}, U_{t t}, V_{t t}, U_{x x}, V_{x x}, \cdots\right)=0,
\end{aligned}
$$

which has a second-order Lagrangian $L$; that is, ((8a) and (8b)) are equivalent to the Euler-Lagrange equations:

$$
\frac{\delta L}{\delta U}=0, \quad \frac{\delta L}{\delta V}=0 .
$$

Definition 1. The vector field $X$, of the form (3), is called a Noether operator corresponding to a Lagrangian $L$ of ((8a) and ( $8 b))$ if

$$
X^{[2]}(L)+\left\{D_{t}\left(\xi^{1}\right)+D_{x}\left(\xi^{2}\right)\right\} L=D_{t}\left(B^{1}\right)+D_{x}\left(B^{2}\right)
$$

for some gauge functions $B^{1}(t, x, U, V)$ and $B^{2}(t, x, U, V)$.

We recall the following theorem.

Theorem 2 (Noether [17]). If X, as given in (3), is a Noether point symmetry generator corresponding to a Lagrangian $L$ of (8a) and (8b), then the vector $T=\left(T^{1}, T^{2}\right)$ with components,

$$
\begin{aligned}
T^{1}= & \xi^{1} L+W^{1} \frac{\delta L}{\partial U_{t}}+W^{2} \frac{\delta L}{\partial V_{t}}-D_{t}\left(W^{1}\right) \frac{\delta L}{\partial U_{t t}} \\
& -D_{t}\left(W^{2}\right) \frac{\delta L}{\partial V_{t t}}-B^{1}, \\
T^{2}= & \xi^{2} L+W^{1} \frac{\delta L}{\partial U_{x}}+W^{2} \frac{\delta L}{\partial V_{x}}-D_{x}\left(W^{1}\right) \frac{\delta L}{\partial U_{x x}}
\end{aligned}
$$

$$
-D_{x}\left(W^{2}\right) \frac{\delta L}{\partial V_{x x}}-B^{2},
$$

is a conserved vector for $((8 \mathrm{a})$ and $(8 \mathrm{~b}))$ associated with the operator $X$, where $W^{1}=\eta^{1}-U_{t} \xi^{1}-U_{x} \xi^{2}$ and $W^{2}=\eta^{2}-V_{t} \xi^{1}-$ $V_{x} \xi^{2}$ are the Lie characteristics functions.

\section{Conservation Laws of System ((2a) and (2b))}

Consider the variable coefficient variant Boussinesq system ((2a) and (2b)); namely,

$$
\begin{gathered}
u_{t}+\alpha(t) v_{x}+\beta(t) u u_{x}=0, \\
v_{t}+\beta(t) u v_{x}+\beta(t) v u_{x}+\psi(t) u_{x x x}=0 .
\end{gathered}
$$

Here we note that the system $((2 \mathrm{a})$ and $(2 \mathrm{~b}))$ does not admit a Lagrangian. Nevertheless, we can transform the system ((2a) and (2b)) into a variational form by setting $u=U_{x}$ and $v=V_{x}$. Thus, the system $((2 \mathrm{a})$ and $(2 \mathrm{~b}))$, with this transformation, becomes a fourth-order system, namely

$$
\begin{gathered}
U_{t x}+\alpha(t) V_{x x}+\beta(t) U_{x} U_{x x}=0, \\
V_{t x}+\beta(t) U_{x} V_{x x}+\beta(t) V_{x} U_{x x}+\psi(t) U_{x x x x}=0
\end{gathered}
$$


and has a second-order Lagrangian given by

$$
L=\frac{1}{2}\left[\psi(t) U_{x x}^{2}-\alpha(t) V_{x}^{2}-\beta(t) U_{x}^{2} V_{x}-V_{t} U_{x}-U_{t} V_{x}\right]
$$

Substituting the value of $L$ from (14) to (1) and splitting with respect to the derivatives of $u$ and $v$ yield the linear overdetermined system of PDEs; namely

$$
\begin{gathered}
\xi_{U}^{1}=0, \quad \xi_{V}^{1}=0, \quad \xi_{V}^{2}=0, \quad \xi_{U}^{2}=0, \\
\xi_{x}^{1}=0, \quad \eta_{U}^{2}=0, \quad \eta_{V}^{1}=0, \quad \eta_{x}^{2}=0, \\
2 \beta(t) \eta_{U}^{1}+\beta(t) \xi_{t}^{1}+\beta(t) \eta_{V}^{2}+\beta^{\prime}(t) \xi^{1}-2 \beta(t) \xi_{x}^{2}=0, \\
\psi^{\prime}(t) \xi^{1}+2 \psi(t) \eta_{U}^{1}-3 \psi(t) \xi_{x}^{2}+\psi(t) \xi_{t}^{1}=0, \quad \eta_{U U}^{1}=0, \\
\alpha^{\prime}(t) \xi^{1}+2 \alpha(t) \eta_{V}^{2}-\alpha(t) \xi_{x}^{2}+\alpha(t) \xi_{t}^{1}=0, \\
\eta_{x x}^{1}=0, \quad \eta_{V}^{2}+\eta_{U}^{1}=0, \quad \xi_{t}^{2}-\beta(t) \eta_{x}^{1}=0, \\
-\eta_{t}^{2}=2 B_{U}^{2}, \quad B_{U}^{1}=0, \quad-\eta_{t}^{1}=2 B_{V}^{2}, \\
-\eta_{x}^{1}=2 B_{V}^{1}, \quad B_{t}^{1}+B_{x}^{2}=0 .
\end{gathered}
$$

After some tedious and lengthy calculations, the above system yields

$$
\begin{gathered}
\xi^{1}=a(t), \\
\xi^{2}=c_{1} x+c_{2} \int \beta(t) d t+c_{3}, \\
\eta^{1}=h(t) U+c_{2} x+k(t), \\
\eta^{2}=-h(t) V+m(t), \\
B^{1}=-\frac{c_{2}}{2} V+z(t, x), \\
B^{2}=-\frac{1}{2} h^{\prime}(t) U V-\frac{1}{2} m^{\prime}(t) U-\frac{1}{2} k^{\prime}(t) V+w(t, x), \\
z_{t}+w_{x}=0, \\
\beta(t) h(t)+\beta(t) a^{\prime}(t)+\beta^{\prime}(t) a(t)-2 c_{1} \beta(t)=0, \\
2 \psi(t) h(t)+\psi^{\prime}(t) a(t)+\psi(t) a^{\prime}(t)-3 c_{1} \psi(t)=0, \\
2 \alpha(t) h(t)-\alpha(t) a^{\prime}(t)-\alpha^{\prime}(t) a(t)+c_{1} \alpha(t)=0 .
\end{gathered}
$$

The analysis of (17), (18), and (19) prompts the following two cases.

Case 1. $\alpha(t), \beta(t)$, and $\psi(t)$ are arbitrary but not of the form contained in Case 2.
In this case we obtain four Noether point symmetries. These are given below together with their corresponding gauge functions:

$$
\begin{gathered}
X_{1}=\frac{\partial}{\partial x}, \quad B^{1}=z, \quad B^{2}=w, \quad z_{t}+w_{x}=0, \\
X_{2}=k(t) \frac{\partial}{\partial U}, \quad B^{1}=z, \quad B^{2}=-\frac{1}{2} k^{\prime}(t) V+w, \\
z_{t}+w_{x}=0, \\
X_{3}=m(t) \frac{\partial}{\partial V}, \quad B^{1}=-\frac{1}{2} m^{\prime}(t) U+z, \quad B^{2}=w, \\
z_{t}+w_{x}=0,
\end{gathered}
$$

$$
\begin{gathered}
X_{4}=\int \beta(t) d t \frac{\partial}{\partial x}+x \frac{\partial}{\partial U}, \quad B^{1}=-\frac{1}{2} V+z, \\
B^{2}=w, \quad z_{t}+w_{x}=0 .
\end{gathered}
$$

Invoking Theorem 2, the four nontrivial conserved vectors associated with these four Noether point symmetries are, respectively,

$$
T_{1}^{1}=u v-z
$$

$$
T_{1}^{2}=\frac{\alpha(t)}{2} v^{2}-\frac{\psi(t)}{2} u_{x}^{2}+\psi(t) u u_{x x}+\beta(t) u^{2} v-w
$$

$$
\begin{gathered}
T_{2}^{1}=-k(t) v+D_{x}\left(\frac{k(t)}{2} \int v d x\right)-z \\
T_{2}^{2}=-\beta(t) k(t) u v-\psi(t) k(t) u_{x x}+k^{\prime}(t) \int v d x \\
-D_{t}\left(\frac{k(t)}{2} \int v d x\right)-w \\
T_{3}^{2}=-m(t) u+D_{x}\left(\frac{m(t)}{2} \int u d x\right)-z \\
-\alpha(t) m(t) v-\frac{1}{2} \beta(t) m(t) u^{2}+m^{\prime}(t) \int u d x \\
T_{4}^{1}=D_{t}\left(\frac{m(t)}{2} \int u d x\right)-w ; \\
T_{4}^{2}=\frac{\alpha(t)}{2} v^{2} \int \beta(t) d t-\frac{\psi(t)}{2} u_{x}^{2} \int \beta(t) d t \\
+\beta(t) x u v-\psi(t) x u_{x x}+\beta(t) u^{2} v \int \beta(t) d t \\
+\psi(t) u u_{x x} \int \beta(t) d t+\psi(t) u_{x}-D_{t}\left(\frac{x}{2} \int v d x\right)-w .
\end{gathered}
$$


From the above we observe that the conserved vector (24)(25) is a local conserved vector. In (30)-(31) one can see that the nonlocal part within the parenthesis gives the trivial part of the conserved vector and therefore can be set to zero. Thus, the conserved vector (30)-(31) is a local conserved vector. It is also interesting to notice that the conserved vectors (26)-(27) and (28)-(29) for $k(t)=1, z(x, t)=0, w(x, t)=0$, and $m(t)=$ 1 yield the local conserved vectors:

$$
\begin{array}{ll}
T_{5}^{1}=v, & T_{5}^{2}=\beta(t) u v+\psi(t) u_{x x} ; \\
T_{6}^{1}=u, & T_{6}^{2}=\alpha(t) v+\frac{1}{2} \beta(t) u^{2} .
\end{array}
$$

Remark 3. We note that for arbitrary values of $k(t)$ and $m(t)$ infinitely many nonlocal conservation laws exist for the variable coefficient variant Boussinesq system.

Case 2. $\alpha(t)=\alpha_{1}, \beta(t)=\beta_{1}$, and $\psi(t)=\psi_{1}$, where $\alpha_{1}, \beta_{1}$, and $\psi_{1}$ are constants.

This case gives us five Noether point symmetries, namely, $X_{1}, X_{2}$, and $X_{3}$, given by the generators (20)-(22) and $X_{4}, X_{5}$ given by

$$
\begin{gathered}
X_{4}=\frac{\partial}{\partial t}, \quad B^{1}=z, \quad B^{2}=w, \quad z_{t}+w_{x}=0 \\
X_{5}=\beta_{1} t \frac{\partial}{\partial x}+x \frac{\partial}{\partial U}, \quad B^{1}=-\frac{1}{2} V+z \\
B^{2}=w, \quad z_{t}+w_{x}=0 .
\end{gathered}
$$

The application of Theorem 2, due to Noether, gives the five nontrivial conserved vectors:

$$
\begin{gathered}
T_{1}^{1}=u v-z, \\
T_{1}^{2}=\frac{1}{2} \alpha_{1} v^{2}-\frac{1}{2} \psi_{1} u_{x}^{2}+\psi_{1} u u_{x x}+\beta_{1} u^{2} v-w ; \\
T_{2}^{1}=-k(t) v+D_{x}\left(\frac{k(t)}{2} \int v d x\right)-z, \\
T_{2}^{2}=-\beta_{1} k(t) u v-\psi_{1} k(t) u_{x x}+k^{\prime}(t) \int v d x \\
-D_{t}\left(\frac{k(t)}{2} \int v d x\right)-w ; \\
T_{3}^{1}=-m(t) u+D_{x}\left(\frac{m(t)}{2} \int u d x\right)-z, \\
T_{3}^{2}=-\alpha_{1} m(t) v-\frac{1}{2} \beta_{1} m(t) u^{2}+m^{\prime}(t) \int u d x \\
-D_{t}\left(\frac{m(t)}{2} \int u d x\right)-w,
\end{gathered}
$$

$$
\begin{gathered}
T_{4}^{1}=\frac{1}{2} \psi_{1} u_{x}^{2}-\frac{1}{2} \alpha_{1} v^{2}-\frac{1}{2} \beta_{1} u^{2} v-z \\
T_{4}^{2}=-\psi_{1} u_{t} u_{x}+\left(\alpha_{1} v+\frac{1}{2} \beta_{1} u^{2}\right) \int v_{t} d x \\
+\left(\beta_{1} u v+\psi_{1} u_{x x}\right) \int u_{t} d x+\int u_{t} d x \int v_{t} d x-w \\
T_{5}^{1}=\beta_{1} u v t-x v+D_{x}\left(\frac{x}{2} \int v d x\right)-z \\
T_{5}^{2}=\frac{1}{2} \alpha_{1} \beta_{1} v^{2} t-\frac{1}{2} \psi_{1} \beta_{1} u_{x}^{2} t-\beta_{1} x u v-\psi_{1} x u_{x x}+\beta_{1} u^{2} v t \\
+\psi_{1} u u_{x x} t+\psi_{1} u_{x}-D_{t}\left(\frac{x}{2} \int v d x\right)-w
\end{gathered}
$$

respectively, corresponding to the above five Noether point symmetries. We note that in this case we obtain an extra Noether operator and hence an extra conserved vector, which is given by (40)-(41).

Remark 4. When $\alpha_{1}=\beta_{1}=\psi_{1}=1$, we recover the results obtained in [8].

\section{Concluding Remarks}

In this paper we studied the variable coefficient variant Boussinesq system $((2 a)$ and $(2 b))$. This system does not have a Lagrangian. Therefore we converted it to a fourth-order system ((13a) and (13b)) which has a Lagrangian. Thereafter, we utilized the Noether's theorem to construct the conservation laws of system ((13a) and (13b)). Finally, by reverting back to our original variables $u$ and $v$ we constructed the conservation laws for the third-order variable coefficient variant Boussinesq system. The conservation laws obtained consisted of infinite number of local and nonlocal conserved vectors.

\section{Conflict of Interests}

The authors declare that there is no conflict of interests regarding the publication of this paper.

\section{Acknowledgment}

Ben Muatjetjeja would like to thank the Faculty Research Committee of FAST, North-West University, Mafikeng Campus, South Africa, for its continuing support.

\section{References}

[1] J. Boussinesq, "Thorie de l'intumescence liquide, applele onde solitaire ou de translation, se propageant dans un canal rectangulaire," Comptes Rendus de L'Academie des Sciences, vol. 72, pp. 755-759, 1871.

[2] R. L. Sachs, "On the integrable variant of the Boussinesq system: Painlevé property, rational solutions, a related many-body system, and equivalence with the AKNS hierarchy," Physica D, vol. 30, no. 1-2, pp. 1-27, 1988. 
[3] E. Fan and Y. C. Hon, "A series of travelling wave solutions for two variant Boussinesq equations in shallow water waves," Chaos, Solitons \& Fractals, vol. 15, no. 3, pp. 559-566, 2003.

[4] M. Wang, "Solitary wave solution variant Boussinesq equations travelling wave solutions for two variant Boussinesq equations in shallow water waves," Chaos, Solitons \& Fractals, vol. 15, pp. 559-566, 2003.

[5] Z. Fu, S. Liu, and S. Liu, "New transformations and new approach to find exact solutions to nonlinear equations," Physics Letters A, vol. 299, no. 5-6, pp. 507-512, 2002.

[6] J. F. Zhang, "Multi-solitary wave solutions for variant Boussinesq equations and Kupershmidt equations," Applied Mathematics and Mechanics, vol. 21, no. 2, pp. 171-175, 2000.

[7] G.-Q. Xu, Z.-B. Li, and Y.-P. Liu, "Exact solutions to a large class of nonlinear evolution equations," The Chinese Journal of Physics, vol. 41, no. 3, pp. 232-241, 2003.

[8] R. Naz, F. M. Mahomed, and T. Hayat, "Conservation laws for third-order variant Boussinesq system," Applied Mathematics Letters, vol. 23, no. 8, pp. 883-886, 2010.

[9] P. D. Lax, "Integrals of nonlinear equations of evolution and solitary waves," Communications on Pure and Applied Mathematics, vol. 21, no. 5, pp. 467-490, 1968.

[10] T. B. Benjamin, “The stability of solitary waves," Proceedings of the Royal Society A, vol. 328, no. 1573, pp. 153-183, 1972.

[11] R. J. Knops and C. A. Stuart, "Quasiconvexity and uniqueness of equilibrium solutions in nonlinear elasticity," Archive for Rational Mechanics and Analysis, vol. 86, no. 3, pp. 233-249, 1984.

[12] R. J. LeVeque, Numerical Methods for Conservation Laws, Birkhuser, Basel, Switzerland, 2nd edition, 1992.

[13] E. Godlewski and P.-A. Raviart, Numerical Approximation of Hyperbolic Systems of Conservation Laws, vol. 118 of Applied Mathematical Sciences, Springer, Berlin, Germany, 1996.

[14] A. Sjöberg, "Double reduction of PDEs from the association of symmetries with conservation laws with applications," Applied Mathematics and Computation, vol. 184, no. 2, pp. 608-616, 2007.

[15] A. H. Bokhari, A. Y. Al-Dweik, A. H. Kara, F. M. Mahomed, and F. D. Zaman, "Double reduction of a nonlinear $(2+1)$ wave equation via conservation laws," Communications in Nonlinear Science and Numerical Simulation, vol. 16, no. 3, pp. 1244-1253, 2011.

[16] G. L. Caraffini and M. Galvani, "Symmetries and exact solutions via conservation laws for some partial differential equations of mathematical physics," Applied Mathematics and Computation, vol. 219, no. 4, pp. 1474-1484, 2012.

[17] E. Noether, "Invariante Variationsprobleme," Nachrichten von der Königlichen Gesellschaft der Wissenschaften zu Göttingen, vol. 2, pp. 235-257, 1918.

[18] N. H. Ibragimov, Ed., CRC Handbook of Lie Group Analysis of Differential Equations, vol. 1-3, CRC Press, Boca Raton, Fla, USA, 1994-1996.

[19] R. Naz, D. P. Mason, and F. M. Mahomed, "Conservation laws and conserved quantities for laminar two-dimensional and radial jets," Nonlinear Analysis-Real World Applications, vol. 10, no. 5, pp. 2641-2651, 2009. 


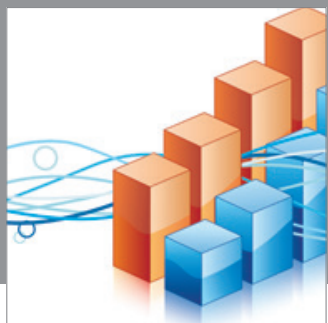

Advances in

Operations Research

mansans

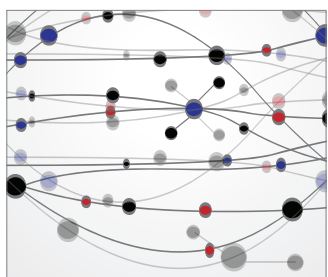

The Scientific World Journal
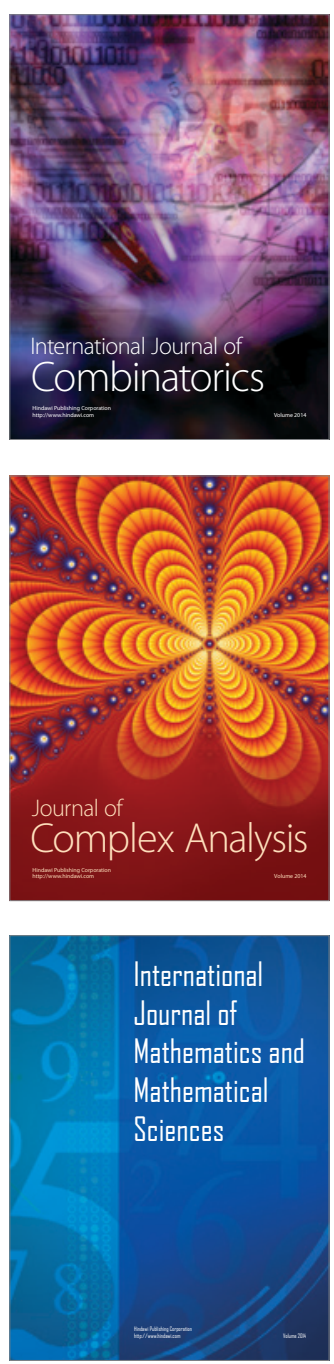
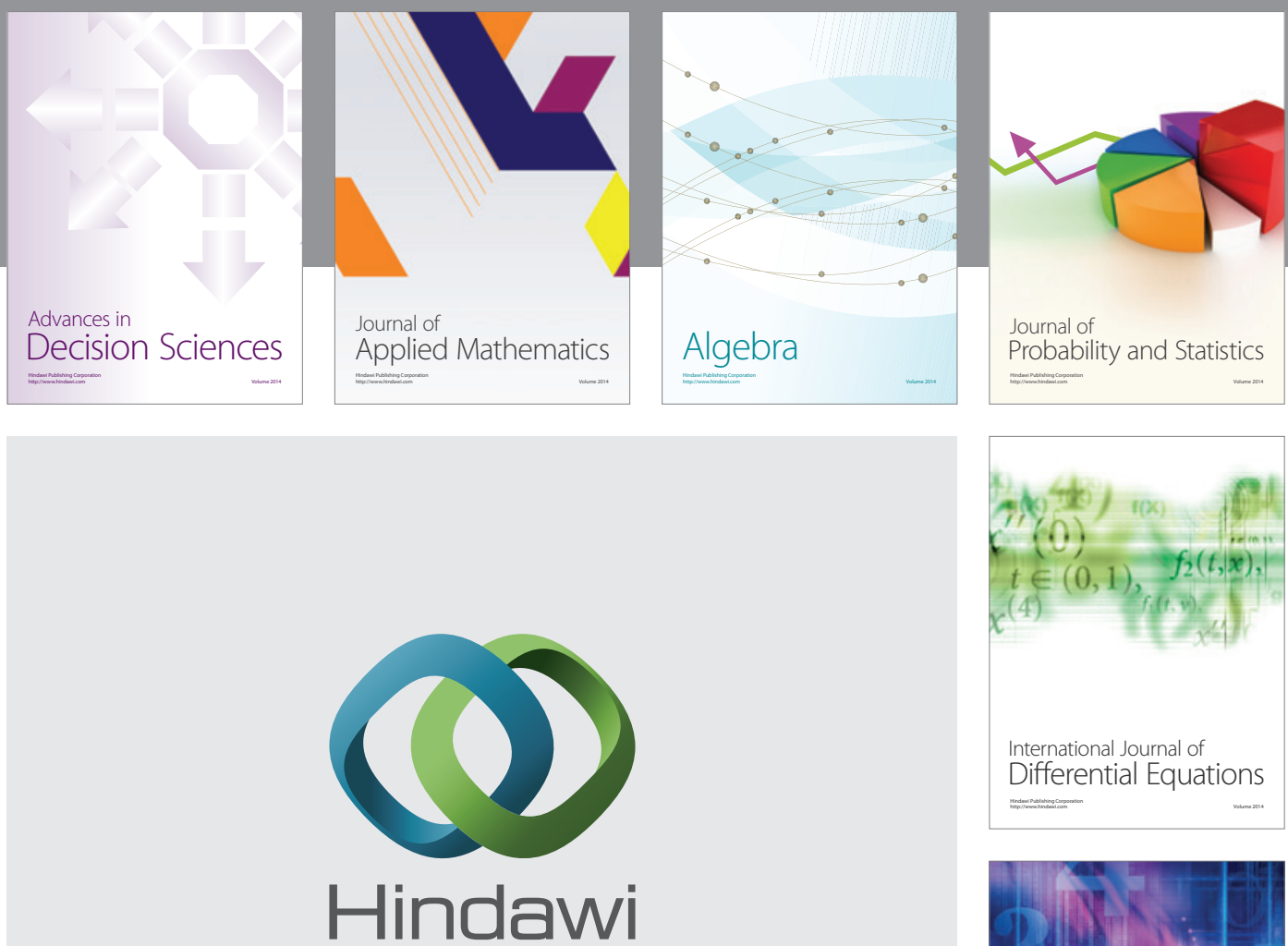

Submit your manuscripts at http://www.hindawi.com
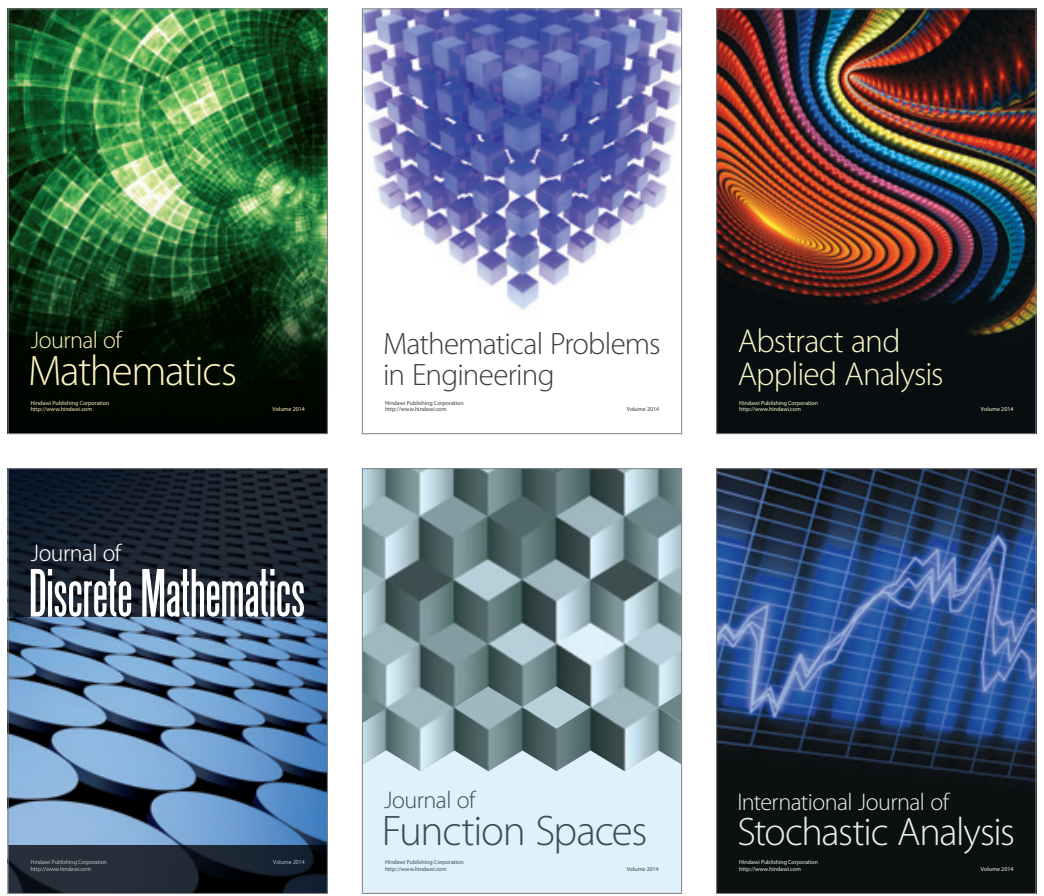

Journal of

Function Spaces

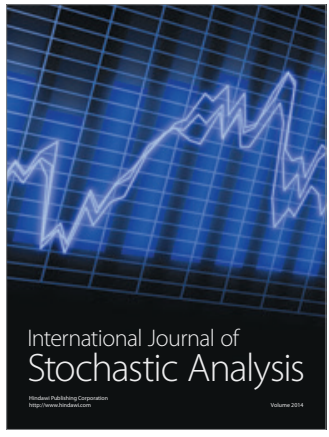

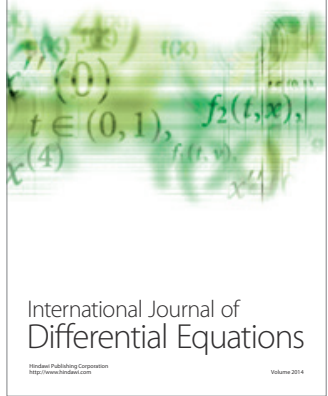
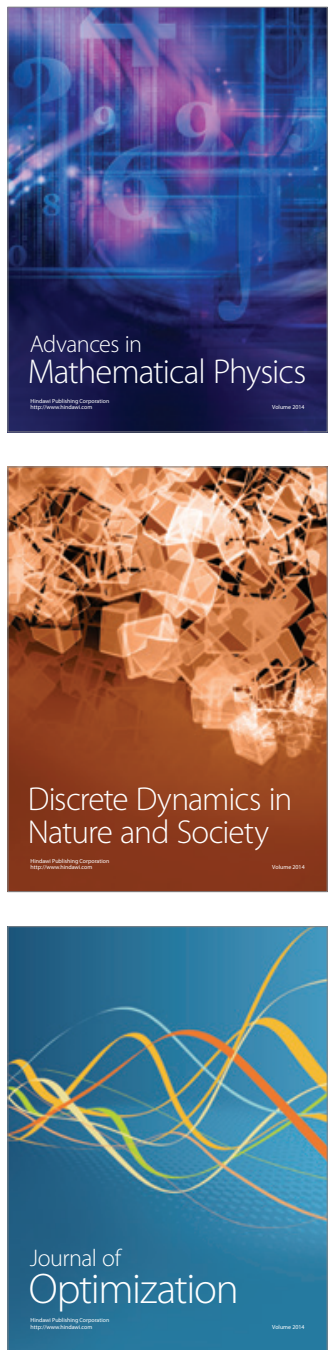